\title{
On the origin of deletions and point mutations in Duchenne muscular dystrophy: most deletions arise in oogenesis and most point mutations result from events in spermatogenesis
}

\author{
T Grimm, G Meng, S Liechti-Gallati, T Bettecken, C R Müller, B Müller
}

\begin{abstract}
We present the results of a study of the rate and origin of mutations in Duchenne muscular dystrophy (DMD). Depending on the type of mutation (deletion/duplication or point mutation) present in the patient, there are widely varying ratios of male to female mutation rates. In deletions, the male mutation rate is only $30 \%$ of the female one. In non-deletional/ non-duplicational mutations (presumably containing a high proportion of point mutations) the male mutation rate is at least $2 \cdot 2$ as high as the female one and probably much higher. Allowing for the presence of autosomal recessive phenocopies we find that $k$ in non-deletional/ non-duplicational mutations is $\mathbf{4 0 \cdot 3}$.

These findings mean that the vast majority of deletions arise in oogenesis, while most point mutations stem from spermatogenesis. Previous investigations have shown that in other diseases and genes, most notably haemophilia $B$ and $A$, but also the ZFY and ZFX genes, the male mutation rate for point mutations tends to be higher than the female one. Our results can be seen as a confirmation of this for the special case of DMD.

The influence on risk figures is considerable. As an example, the risk of the mother of an isolated case of DMD without an apparent structural anomaly of the gene of being a carrier increases from $67 \%$ to at least $76 \%$. Given the estimate of 40.3 for $k$, allowing for the presence of autosomal recessive phenocopies mentioned above, it increases even further to $\mathbf{9 8 \%}$. However, as confidence intervals are still large, more data are needed to improve the estimates. Germinal mosaicism in this context is discussed.
\end{abstract}

(f Med Genet 1994;31:183-186)

The estimation of the ratio of male and female mutation rates in Duchenne muscular dystrophy (DMD), an X linked recessive, genetically lethal disorder, has been a matter of some interest, both for scientific reasons and for genetic counselling, as this ratio, which will be called $\mathrm{k}$ in the following, has, among others, implications for risk calculation. It should also give some insight into the origin of DMD mutations. We present the results of a retrospective study, analysed by the indirect method of Müller and Grimm, ${ }^{1}$ in order to estimate $k$ for different types of mutations separately. A large collaborative study ${ }^{2}$ using the same methodology has shown that the overall mutation rates for mutations causing DMD are about equal in males and females. The results of the present analysis not only give indications of the ratio of mutation rates, but also the origin of mutations.

\section{Material and methods}

\section{FAMILIES}

Data were collected at the genetic service of the Department of Human Genetics, University of Würzburg. To be included in the study, the following criteria had to be met by the families studied. (1) There had to be a definite diagnosis of DMD in the index patient. (2) Families had to be referred for carrier/prenatal diagnosis. (3) A blood sample from the index patient had to be available for deletion screening. There were 280 families fulfilling these three criteria.

Intragenic recombinations in families were excluded and a correction for the possible bias thus introduced was performed as described by Müller et al. ${ }^{2}$ Blood samples from both the mother and the two maternal grandparents had to be available, too, or, if this was not the case, families were used only if the missing haplotypes could be reconstructed without ambiguities from the genotypes of the patient, his mother, and one of the grandparents. No information from other family members was used to avoid any bias for familial cases.

In a total of 117 families the grandparental origin of the mutated haplotype could be determined. These families were thus informative for the study. A duplication was found in six of these families. Detailed analysis of duplications was not pursued further because of the small numbers.

The distribution of mutation types in these families is given in table 1 . It should be noted that what is called a "point mutation" in the text probably consists of a mixture of different mutation types, including point mutations, small deletions, and small duplications. They do, however, share the common feature of not being detectable on deletion screening. We did not find significantly different prevalences of proximal and distal deletions in familial versus 
Table 1 Distribution of families

\begin{tabular}{lcccc}
\hline & Deletion & Duplication & Point mutation & Total \\
\hline Sporadics cases & 114 & 8 & 76 & 198 \\
$?$ & 72 & 4 & 38 & 114 \\
GMH & 26 & 2 & 13 & 41 \\
GPH & 16 & 2 & 24 & 42 \\
Affected sibs & 22 & 2 & 14 & 38 \\
? & 17 & 11 & 1 & 6 \\
GMH & 4 & 1 & 2 & 4 \\
GPH & 1 & 0 & 10 & 44 \\
Extended family history & 21 & 0 & 13 & 20 \\
? & 10 & 0 & 0 & 24 \\
GMH & 11 & 10 & 113 & 0 \\
GPH & 0 & 4 & 59 & 280 \\
All family configurations & 157 & 3 & 27 & 71 \\
? & 99 & 3 & & 46 \\
GMH & 11 & 17 & & \\
GPH & 17 & & \\
\hline
\end{tabular}

? Grandparental haplotype could not be determined.

$\mathrm{GMH}=$ grandmaternal haplotype

$\mathrm{GPH}=$ grandpaternal haplotype.

sporadic cases, contrary to the findings of Passos-Bueno et al, ${ }^{3}$ so we did not consider them separately (data not shown).

\section{Ascertainment}

As described above, a request for carrier or prenatal diagnosis or both was one of the selection criteria. While avoiding a possible bias in favour of cases diagnosed through deletion screening, this could possibly lead to bias for a certain family configuration. In a recent Dutch study with near complete ascertainment, ${ }^{4}$ sporadic cases accounted for $62 \%$ of the families studied, affected sibs for $16 \%$, and cases with an extended family history for $22 \%$. In our sample these proportions were significantly different $\left(\chi^{2} 9.95,2 \mathrm{df}, \mathrm{p}<0.01\right)$ with most of the difference resulting from an excess of sporadic cases $(71 \%)$. This may have been caused by our selection criteria demanding the index patient to be available for testing. As this finding would bias our estimate of $\mathrm{k}$ we corrected the respective proportions of sporadic cases, cases with affected sibs, and cases with an extended family history in our sample to conform with the Dutch results.

DELETION SCREENING

Deletion screening was done by standard methods. $^{5}$

\section{HAPLOTYPE ANALYSIS}

Haplotype analysis was done using a total of 15 RFLPs partly within or close to either end of the gene. Recombinant haplotypes were excluded from the analysis and corrected for, as mentioned above.

\section{ANALYSIS}

First, we analysed the proportions of grandmaternal and grandpaternal haplotypes in patients with deletions and presumed point mutations by means of a two by two contingency table (tables $2 \mathrm{~A}$ and B) after correcting for ascertainment bias as described above.

In a second step we estimated the ratio $k$ of male to female mutation rates both for deletions and point mutations. This approach was
Table 2 Distribution of grandparental haplotypes

\begin{tabular}{lll}
\hline & Deletion & Point mutation \\
\hline (A) & & \\
GMH & 41 & 27 \\
GPH & 17 & 26 \\
(B) & & \\
GMH & 42.44 & 26.04 \\
GPH & 15.56 & 26.96 \\
(C) & & \\
GMH & 42.44 & 23.74 \\
GPH & 15.56 & 22.64 \\
\hline
\end{tabular}

GMH = grandmaternal haplotype

$\mathrm{GPH}=$ grandpaternal haplotype.

based on the indirect method of Müller and Grimm $^{1}$ with the correction introduced and described in detail by Müller et al. ${ }^{2}$

The method used may be briefly outlined as follows. The mutation present in a DMD patient may have arisen de novo in either the patient himself (with a probability of the female mutation rate $\mu$ on the grandpaternal $X$ chromosome and with the same probability on the grandmaternal $\mathrm{X}$ chromosome) or the mother of the patient (on either the paternal X chromosome with probability $\mathrm{v}$, the male $\mathrm{mu}$ tation rate, or the maternal $\mathrm{X}$ chromosome with probability $\mu$ ). The third possibility is that the grandmother of the patient is already a carrier, which according to Haldane's equilibrium $^{6}$ has a probability of $2(\mu+v)$.

Then the distribution of grandmaternal and grandpaternal haplotypes is determined only by the ratio $k$ of the male and female mutation rates. The proportion of grandpaternal haplotypes among all DMD index patients is given by

\section{$\frac{\mathrm{GPH}}{(\mathrm{GMH}+\mathrm{GPH})}=\frac{\mathrm{v}+\mu}{(4 \mu+2 \mathrm{v})}$, as can be derived} from the above.

Then $\mathrm{k}$, which is $\mathrm{v} / \mu$, becomes

$$
\mathrm{k}=\frac{3 \mathrm{GPH}-\mathrm{GMH}}{\mathrm{GMH}-\mathrm{GPH}} .
$$

A factor $\mathrm{r}$ has to be introduced to allow for a possible bias owing to the omission of cases with intragenic recombinations:

$$
\mathrm{k}=\frac{(3-\mathrm{r}) \mathrm{GPH}-(1-\mathrm{r}) \mathrm{GMH}}{\mathrm{GMH}-\mathrm{GPH}}
$$

with GPH and GMH being the absolute numbers of grandpaternal and grandmaternal haplotypes in our sample and $r$ the probability of intragenic recombination, which we set to $0 \cdot 1$.

We also calculated the proportion of deletions stemming from oogenesis and spermatogenesis, respectively, as well as the corresponding proportions for point mutations.

The proportion of mutations arising in spermatogenesis is, in general, given by

$$
\frac{2 \mathrm{k}}{4+2 \mathrm{k}}
$$


which simplifies to

$$
\frac{\mathrm{k}}{2+\mathrm{k}}
$$

In analogy the proportion of mutations arising in oogenesis is given by

$$
\frac{2}{2+\mathrm{k}}
$$

Applying the above formulae using the ratio $\mathrm{k}$ found in deletions or point mutations, respectively, we find the proportions pertaining to deletions and point mutations.

Germinal mosaicism does not alter our findings very much. Indeed, it can be shown that, following our model of germinal mosaicism published in this journal, ${ }^{7}$ the extra factors needed to introduce germinal mosaicism into the mutation-selection equilibrium for DMD are deeply confounded with the ratio of mutation rates. Incidentally and very nicely, it turns out that the estimate of $\mathrm{k}$ obtained neglecting the possibility of germinal mosaicism is equal to the estimate of $k^{\prime} d$,

$$
\widehat{\mathrm{k}}=\widehat{\mathrm{k}^{\prime} \mathrm{d}}
$$

where $\mathrm{k}^{\prime}$ is again the ratio of male and female mutation rates $\left(k^{\prime}=v / \mu\right)$, and $d$ is a function of the male and female factors needed to introduce germinal mosaicism.

$$
d=\frac{\left(1-g_{f}+g_{f} f_{f}\right)}{\left(1-g_{m}+g_{m} f_{m}\right)}
$$

where $g_{f}$ and $g_{m}$ are the proportions of mutations leading to germinal mosaicism in males and females, respectively. The parameters $f_{f}$ and $f_{m}$ are the proportions of affected germ cells in males and females, respectively. While at first it appears that the confoundment of $k^{\prime}$ and $\mathrm{d}$ may be considered somewhat annoying, it turns out that some major facts in counselling remain untouched, that is, the product $k^{\prime} d$ is equivalent to $\mathrm{k}$ for the calculation of some risks also.

Take as an example the risk for the mother of an affected boy without a previous family history of being a carrier. Neglecting germinal mosaicism, this risk is given as $\frac{1+k}{2+k}$. Allowing for this, the risk is $\frac{1+k^{\prime} d}{2+k^{\prime} d}$, the value of which is identical to $\frac{1+k}{2+k}$, given the relationship (4).

Another problem is the possible presence of autosomal recessive phenocopies (for example, Duchenne-like autosomal recessive muscular dystrophy, MIM 253 700) in our sample. This recessive disease has been estimated to be at most a twentieth as frequent as DMD. ${ }^{8}$ Moreover these autosomal recessive cases may be assumed to be present in our sample exclusively in cases where no mutation in the DMD gene is present in the patient and in the absence of a family history typical of $X$ linkage. If we assume that overall about $5 \%$ of cases with apparent DMD are in fact such phenocopies, then in the subset of sporadics or sib cases with apparent point mutations in our sample, which constitutes 90 of the total 280 patients in table
$1(32 \%)$, we can expect the proportion of phenocopies to be around $16 \%$. The presence of autosomal recessive phenocopies will tend to overestimate $\mathrm{k}$. The possible presence of autosomal recessive phenocopies should be corrected for. It should be noted again that the presence of autosomal recessive phenocopies plays a role only in cases without a detectable structural anomaly of the DMD gene.

\section{Results}

The distribution of grandparental haplotypes conditional on the presence of either a deletion or a point mutation in the index patient is given in table $2 \mathrm{~A}$ (before the correction mentioned in ascertainment), table $2 \mathrm{~B}$ (after the correction), and in table $2 \mathrm{C}$ (after the correction for the presence of $16 \%$ autosomal recessive phenocopies in sporadic or sib cases with supposed point mutations). Table $2 B$, which is the relevant one for the analysis neglecting the presence of autosomal recessive phenocopies, yields a $\chi^{2}$ of $6.77,1 \mathrm{df}, \mathrm{p}<0.01$ (table $2 \mathrm{~A}$ gives a $\chi^{2}$ of $4.55, \mathrm{p}<0.05$ ). Table $2 \mathrm{C}$ gives a $\chi^{2}$ of $5 \cdot 36,1 \mathrm{df}, \mathrm{p}<0.03$.

Our estimate of $k$ in deletions is $0.3(95 \%$ confidence interval $0-2 \cdot 9$ ). In point mutations, assuming absence of autosomal recessive phenocopies, no valid point estimate is reached as, because of small sample variation, GPH is larger than GMH, which yields a negative and thus meaningless estimate of $k$. However, the lower limit of the confidence interval for $k$ in point mutations is $2 \cdot 2$, so we may assume that, neglecting the presence of autosomal recessive phenocopies, the value of $2 \cdot 2$ is a reasonable minimum estimate for $k$ in point mutations. However, $\mathrm{k}$ is probably considerably higher. The difference between $k$ in deletions and $k$ in point mutations appears significant, as suggested by the $\chi^{2}$ in table $2 \mathrm{~B}\left(\chi^{2}\right.$ of $6.77,1 \mathrm{df}$, $\mathrm{p}<0.01)$. The difference between the estimate for $\mathrm{k}$ in deletions and the lower limit for $\mathrm{k}$ in point mutations misses significance applying a likelihood ratio test of the hypothesis of equal $k$ values in both deletions and point mutations against the hypothesis of different values in these types of mutations (likelihood ratio of $\left.5 \cdot 0, \chi^{2} 3.22,1 \mathrm{df}, 0.10<\mathrm{p}<0.05\right)$. It should be noted, however, that this test is probably overly conservative, as we tested the lower limit for the estimate of $\mathrm{k}$ in point mutations owing to the absence of a valid estimate. The overall estimate of $\mathrm{k}$ in all 117 families is $2 \cdot 4$ (95\% confidence interval $0.5-18.9)$, which is in agreement with Müller et $a l^{2}(\mathrm{k}=1 \cdot 14)$. This is not very surprising, however, because of the large overlap of data sets in both studies.

Allowing for the presence of autosomal recessive phenocopies, which is the more realistic assumption, we find an estimate of $k$ in point mutations of 40.3 (95\% confidence interval $2 \cdot 0-\infty)$. The overall estimate of $k$ in both deletions and point mutations becomes 1.83 . The likelihood ratio test as described above is significant (likelihood ratio of $14.6, \chi^{2} 5.36$, $1 \mathrm{df}, \mathrm{p}<<0.03$ ).

Given the above estimates for $k(0.3$ in deletions and $40 \cdot 3$ in point mutations) we find 
that only about $13 \%$ of deletions arise in spermatogenesis and $87 \%$ occur in oogenesis. We also estimate that around $95 \%$ of point mutations arise in spermatogenesis while.only about $5 \%$ result from an event in oogenesis. Disregarding the presence of autosomal recessive phenocopies and assuming the lower limit of $2 \cdot 2$ for $k$ in point mutations, we would estimate that at least $52 \%$ of point mutations arise in spermatogenesis while at most $48 \%$ result from an event in oogenesis.

\section{Discussion}

We present evidence that the ratio of male and female mutation rates in Duchenne muscular dystrophy, although overall they appear to be about equal, differ depending on the type of mutation present. In presumed point mutations, errors in spermatogenesis are the most likely cause of the mutations while the opposite appears true in deletions.

For a variety of other genes it has been shown that for point mutations the male mutation rate is higher than the female one. Shimmin et $a l^{9}$ found a higher rate of nucleotide substitutions in males and estimated $\mathrm{k}$ (for substitutions in the ZFX and XFY genes in humans) to be around 6 . Recently it has been shown for haemophilia B by Ketterling et $a l^{10}$ that the ratio of mutation rates varies with the type of mutation. They found a higher mutation rate in males for single base substitutions (estimate of $k=3.5$ ). In deletions they found a sex ratio of the mutation rates of 1 . In haemophilia A, a study published in this journal in $1991^{11}$ also reported a higher mutation rate in spermatogenesis than in oogenesis. Depending on the method used they estimated $\mathrm{k}$ to be $12 \cdot 1$ or 5.2. Our findings in DMD point mutations show a similar trend to the above results.

Our data concerning deletions are in agreement with a large European study ${ }^{12}$ in which a preferentially maternal origin of deletional mutations was found, although that finding was not significant, possibly because of smaller sample sizes and also the exclusion of certain family types which possibly biased their findings towards a more conservative estimate of $k$.

As far as genetic counselling is concerned, risk calculation depends very much on the ratio $k$. Take as an example the risk of the mother of a sporadic case of being a carrier. If $\mathrm{k}$ were equal to 1 , her risk would be $2 / 3$, which is the figure usually given. Now assume that this patient has a point mutation. His mother's risk of being a carrier is, as a function of $k$, equal to $\frac{1+k}{2+k}$. Thus if $k$ is assumed to be $2 \cdot 2$ her risk increases from $67 \%$ to $76 \%$. It is probably much higher. Allowing for the presence of autosomal recessive phenocopies and using the obtained estimate of 40.3 for $k$, that risk is calculated to be $98 \%$, which is very high. Of course, such figures depend heavily on the value of $k$. The confidence intervals still being large and overlapping, more data are urgently needed to improve our estimates. This paper is dedicated to Professor P E Becker on his 85 th
birthday. The authors would like to acknowledge the cooperation of the DMD families without whom this study would not have been possible. We also acknowledge the help of many friends and colleagues who are not named as authors but whose contributions were very helpful for this study. This study was supported in part by grant GR506/3-1 from the Deutsche Forschungsgemeinschaft to TG and TB.

1 Müller CR, Grimm T. Estimation of the male to female ratio of mutation rates from the segregation of $\mathrm{X}$ chromosomal DNA haplotypes in Duchenne muscular dystrophy families. Hum Genet 1986;74:181-3.

2 Müller B, Dechant C, Meng G, et al. Estimation of the male and female mutation rates in Duchenne muscular dystrophy (DMD). Hum Genet 1992;89:204-6.

3 Passos-Bueno MR, Bakker E, Kneppers ALJ, et al. Dif ferent mosaicism frequencies for proximal and dista Duchenne muscular dystrophy (DMD) mutations indicDuchenne muscular dystrophy (DMD) mutations indic-
ate difference in etiology and recurrence risk. Am $\mathcal{J}$ Hum Genet 1992;51:1150-5.

4 van Essen AJ, Busch HFM, te Meerman GJ, ten Kate LP. Birth and population prevalence of Duchenne muscula dystrophy in the Netherlands. Hum Genet 1992;88:258 66.

5 Koenig M, Hoffman EP, Bertelson CJ, Monaco AP, Feener C, Kunkel LM. Complete cloning of the Duchenn muscular dystrophy (DMD) CDNA and preliminary genomic organization of the DMD gene in normal and affected individuals. Cell 1987;50:509-17.

6 Haldane JBS. Mutation in the sex-linked recessive type of muscular dystrophy. A possible sex difference. Ann Hum Genet 1956;20:344-7.

7 Grimm T, Müller B, Müller CR, Janka M. Theoretical considerations on germinal mosaicism in Duchenne muscular dystrophy. $\mathcal{J}$ Med Genet 1990;27:683-7.

8 Emery AEH. Population frequencies of inherited neuromuscular diseases - a world survey. Neuromusc Dis 1991;1:19-29.

9 Shimmin LC, Chang BHJ, Li WH. Male-driven evolution of DNA sequences. Nature 1993;362:745-7.

10 Ketterling RP, Vielhaber E, Bottema CDK, et al. Germline origins of mutation in families with hemophilia B: the sex ratio varies with the type of mutation. Am $\mathrm{f} \mathrm{Hum}$ Genet 1993;52:152-66.

11 Bröcker-Vriends AHJT, Rosendaal FR, van Houwelingen $\mathrm{JC}$, et al. Sex ratio of the mutation frequencies in haemophilia A: coagulation assays and RFLP analysis. $\mathcal{J} \mathrm{Med}$ Genet 1991;28:672-80.

12 van Essen AJ, Abbs S, Baiget $M$, et al. Parental origin and germline mosaicism of deletions and duplications of the dystrophin gene: a European study. Hum Genet 1992;88:249-57. 